\title{
Atmospheric visibility sensor based on backscattering using correlation coding method
}

\author{
Tomasz Czarnecki · Krzysztof Perlicki • Grzegorz Wilczewski
}

Received: 14 November 2013 / Accepted: 15 May 2014 / Published online: 29 May 2014

(C) The Author(s) 2014. This article is published with open access at Springerlink.com

\begin{abstract}
An new atmospheric visibility sensor is described which uses an empirical relationship between atmospheric visibility and lidar backscatter signals. Specifically, autocorrelation analysis is performed for lidar backscatter signals and the autocorrelation coefficients are shown to be a function of the correlation distance. The slope of this function is then connected to the atmospheric visibility under different weather conditions. It is experimentally demonstrated that this new sensor can reliably estimate the atmospheric visibility in the range of $0-1,200 \mathrm{~m}$.
\end{abstract}

Keywords Atmospheric visibility $\cdot$ Light backscattering $\cdot$ Correlation coding $\cdot$ Lidar

\section{Introduction}

A clear visibility of the surrounding environment is crucial in various types of transportation means. Dense fog or rain developed alongside roadways may considerably decrease the safety of motorists, thus automated detectors and monitoring of the visibility, fog, rain, snow or dust is of the upmost importance. As a feasible variable, atmospheric visibility is difficult to assess. Weather, sun angle, light intensity, darkness adaptation, availability of appropriate visibility targets and individual physical abilities determine the level of human's perception quality of atmospheric conditions. Automated visibility sensors determine the visibility range by measuring light being scattered or by utilization of extinction coefficients, locally over a short range (Siikamaki 2004; Babari et al. 2011). Optical sensors such as

T. Czarnecki · K. Perlicki $(\bowtie) \cdot$ G. Wilczewski

Institute of Telecommunications, Warsaw University of Technology,

Nowowiejska 15/19, 00-665 Warsaw, Poland

e-mail: perlicki@tele.pw.edu.pl

T. Czarnecki

e-mail: t.czarnecki@tele.pw.edu.pl

G. Wilczewski

e-mail: g.wilczewski@tele.pw.edu.pl 
transmissometers or scatterometers were developed to address atmospheric visibility and visual range evaluation. The transmissometer was the first instrument developed to provide a standard for visibility measurement. The device estimates the attenuation of a light beam emitted from a source to a receiver at a certain path length and correlates obtained result with human observations. The light attenuation phenomena is due to scattering or refraction, depending on the utilized medium. However, transmissometers' physical dimensions and long baselines create a multiplicity of disadvantages when performance is considered. Demanding maintenance, high manufacturing costs and small dynamic range with insufficient accuracy discard transmissiometers from being abundantly popular. In case of the scatterometer, measurements are based on the amount of light being scattered by aerosols in an optical volume, observed within a small solid angle. The scatterometer assesses the dispersion of a light beam (Kazovsky 1985; Kemp et al. 2012), nevertheless reliability of a typical scatterometer is reported as questionable (Babari et al. 2011). Atmospheric visibility assessment is also performed with the use of light backscattering phenomenon (Taillade et al. 2008). Therein discussed is the thoroughly performed examination of the analytical model supporting backscattered luminance investigation (with an optical device geometry modeling), and as authors report, a good performance in agreement with experimental results. Another approach towards cruise supporting systems (Hautiere et al. 2008), with respect to the vehicular transportation domain, shows several visual data analysis approaches to enable estimation of the atmospheric visibility in mobile applications, with low margin of error.

In this paper, a brand new method for atmospheric visibility estimation is depicted. Presented sensor setup employs light backscattering effect with use of correlation coding technique. Evaluation of atmospheric visibility range is performed by signal correlation analysis.

\section{Methodology}

2.1 Atmospheric visibility estimation based on backscattering using correlation coding technique

The principle of operation of the designed sensor is based on the analysis of backscattered optical signal. Device's transmitter emits an optical signal into the air where the light is subjected to backward scattering by air particles (for instance: rain drops, snowflakes or fog) and arrives at the receiver where it falls onto a photodetector. The backscattered optical signals within a range from 0 to $250 \mathrm{~m}$ are being analyzed. The principle of operation of the presented sensor is depicted on the schematic diagram in the Fig. 1. Consequently, the setup configuration of the described device is shown in the Fig. 2.

Concerning architectural layout of the presented sensor, it is composed of transmitting and receiving parts. The transmitting module generates pulsed laser light with the wavelength of $808 \mathrm{~nm}$ and the average optical power of $250 \mathrm{~mW}$ (the Bob $808-2 \mathrm{~W}$ laser is utilized). The optical signal generated consists of a $7.5 \mathrm{Mbit} / \mathrm{s}$ binary stream with $2^{9}-1$ length of Pseudo Random Bit Sequence (PRBS). As presented on the Fig. 1, characteristic of emitted radiation is described as nearly parallel as it concentrates in a form of a regular beam with the diameter equal to $50 \mathrm{~mm}$. Transmitting beam intersects with the receiving one at a distance of $250 \mathrm{~m}$ (what further implies the zone taken under consideration in autocorrelation analysis) from the surface of both active parts. In terms of the divergence, the receiving beam parameter is equal to approximately $1^{\prime}$ of the angular spread, while the receiver area diameter is $120 \mathrm{~mm}$. At the receiving end, the avalanche photodiode Hamamatsu C5331-02 (APD module on the Fig. 1) and $808 \mathrm{~nm}$ narrow bandpass optical filter are used. In order to sustain reliable measurements, 
Fig. 1 Schematic diagram of the designed atmospheric visibility sensor
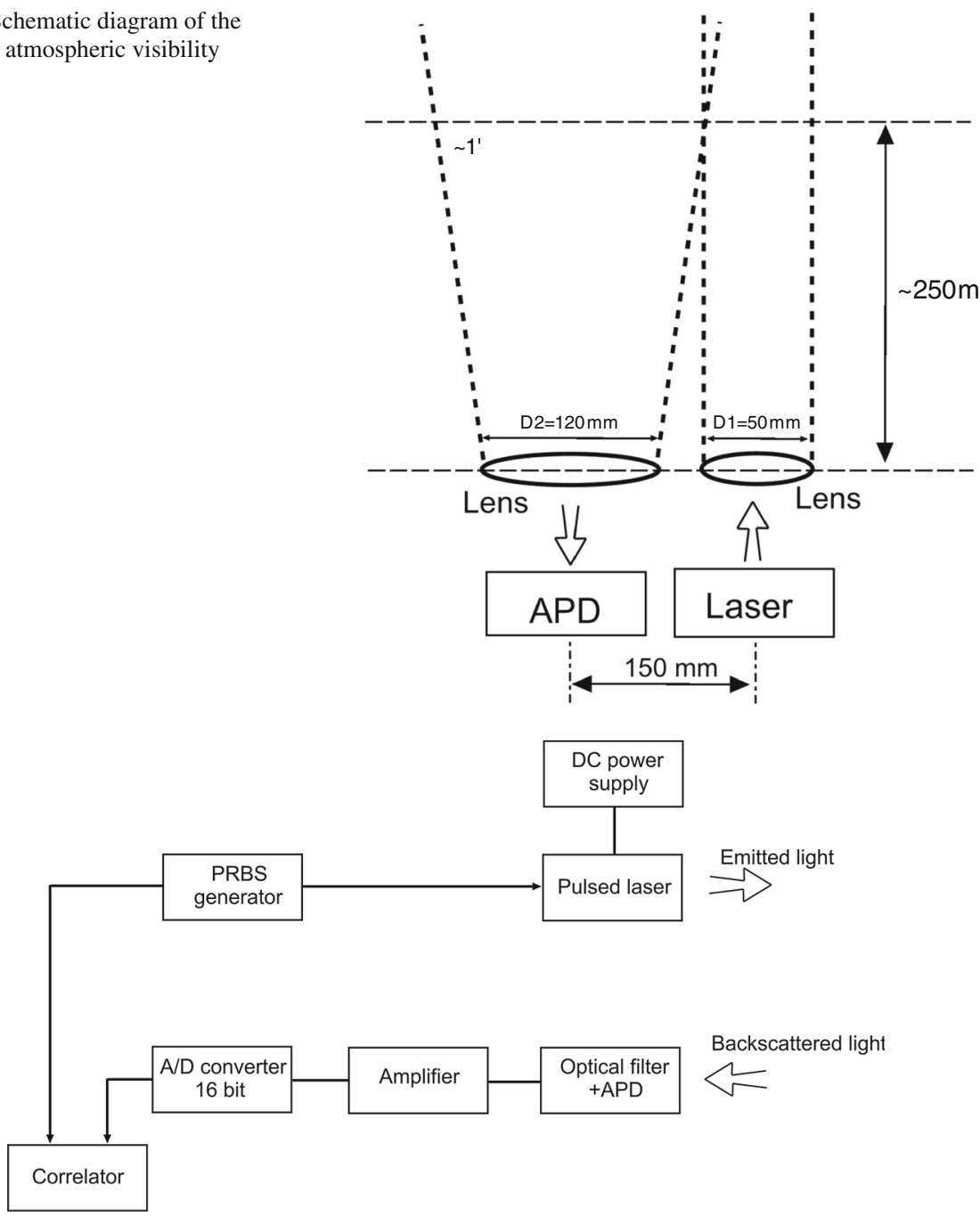

Fig. 2 The atmospheric visibility sensor-setup layout

it is of the upmost importance to prevent the receiver from falling into a saturation mode. Sensor parameters (i.e.: the laser optical power, photodiode active area size, analog to digital converter dynamic range) have been precisely selected, as it is crucial to avoid saturation state of the receiving part. To complete the description of the designed device, the transmitting part consists of the push-pull power driver and synchronization branch towards the wave generator. Receiving section utilizes HYPRES HA103-35 signal amplifier and 16-bit analog to digital converter-ADC 08L060. To organize the workflow, PIC 16F630 microchip is applied to support the control functions, as well as communication with outer devices by means of Universal Serial Bus (USB).

Further, concerning methodology insights, as it is presented on the plots within Fig. 3, stream autocorrelation versus correlation distance for different weather conditions (i.e. atmospheric visibility) is assessed. On the figure, the plots to the left side depict the behavior 

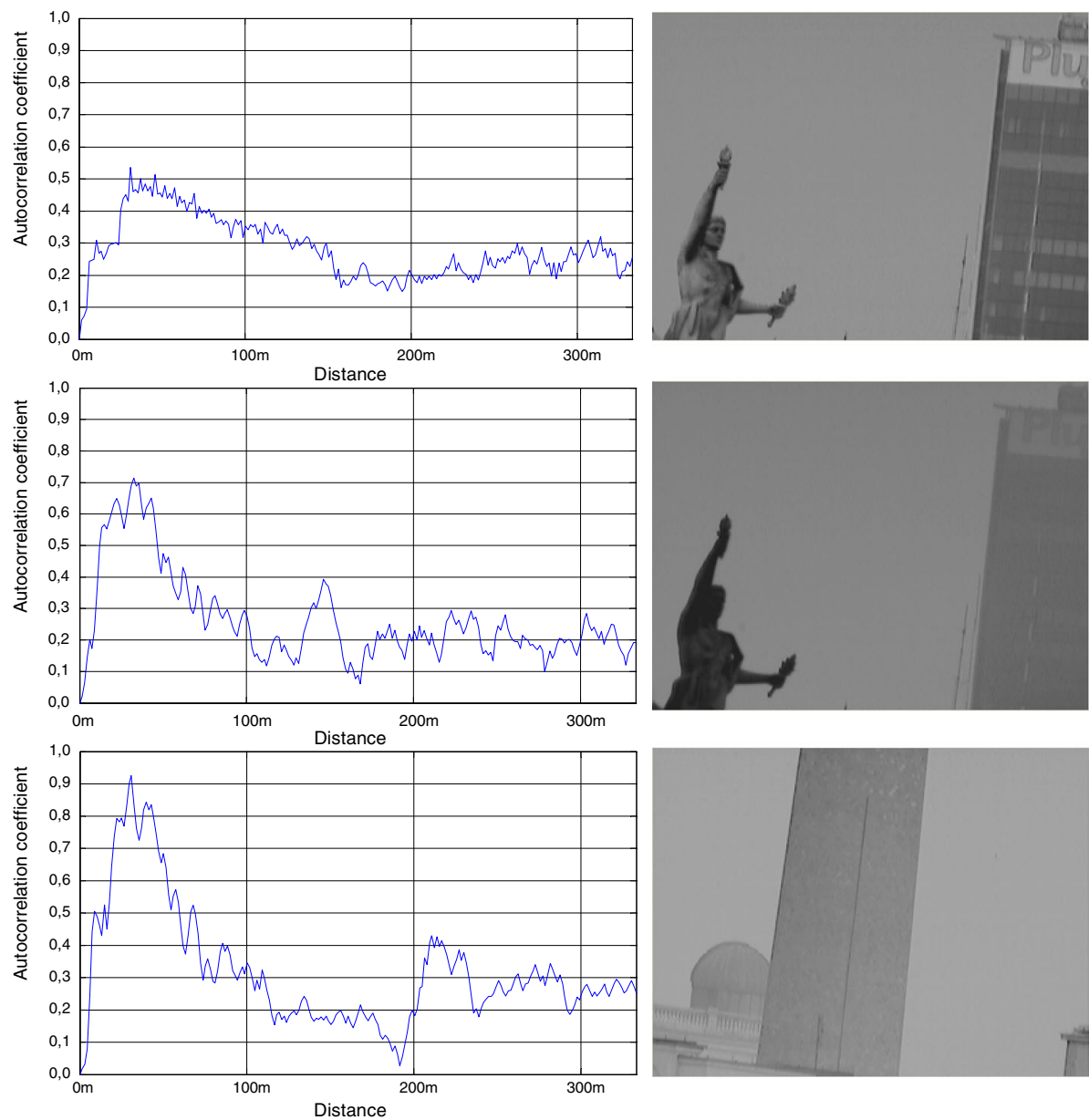

Fig. 3 Autocorrelation coefficient versus correlation distance under different weather conditions (atmospheric visibility)

of the autocorrelation coefficient, as a function of considered distance, reflecting the outdoor atmospheric conditions (as presented by the stacked images to the right). One can see the variety of autocorrelation peaks and respective slopes of resulting falling edges. Achieved results imply relevant relationship between the slope of the autocorrelation peak (graphical assessment presented on the Fig. 4) and atmospheric visibility. Elaborating on the correlation technique itself, signals obtained at the receiving part are processed in the modulo operation implemented on the mesh of correlators (in count and length matching PRBS sequence, however single bit shift operation is performed), executed on the Xilinx Spartan family chip.

Consequently, having calculated the value of correlation distance reflecting the maximum level of the autocorrelation (initial position of the autocorrelation peak-L), one has to determine corresponding values of the autocorrelation index for a set of corresponding distances: $\mathrm{L}+\mathrm{dL}, \mathrm{L}+2 \mathrm{dL}, \mathrm{L}+3 \mathrm{dL}$ and $\mathrm{L}+4 \mathrm{dL}$; where $\mathrm{dL}$ is the distance step of $10 \mathrm{~m}$. Next, approaching the acquisition of the slope of the autocorrelation peak, data fitting algorithm is applied to the values represented by a sequence of points $(\mathrm{A}, \mathrm{B}, \mathrm{C}, \mathrm{D}, \mathrm{E})$, in order to achieve the required 


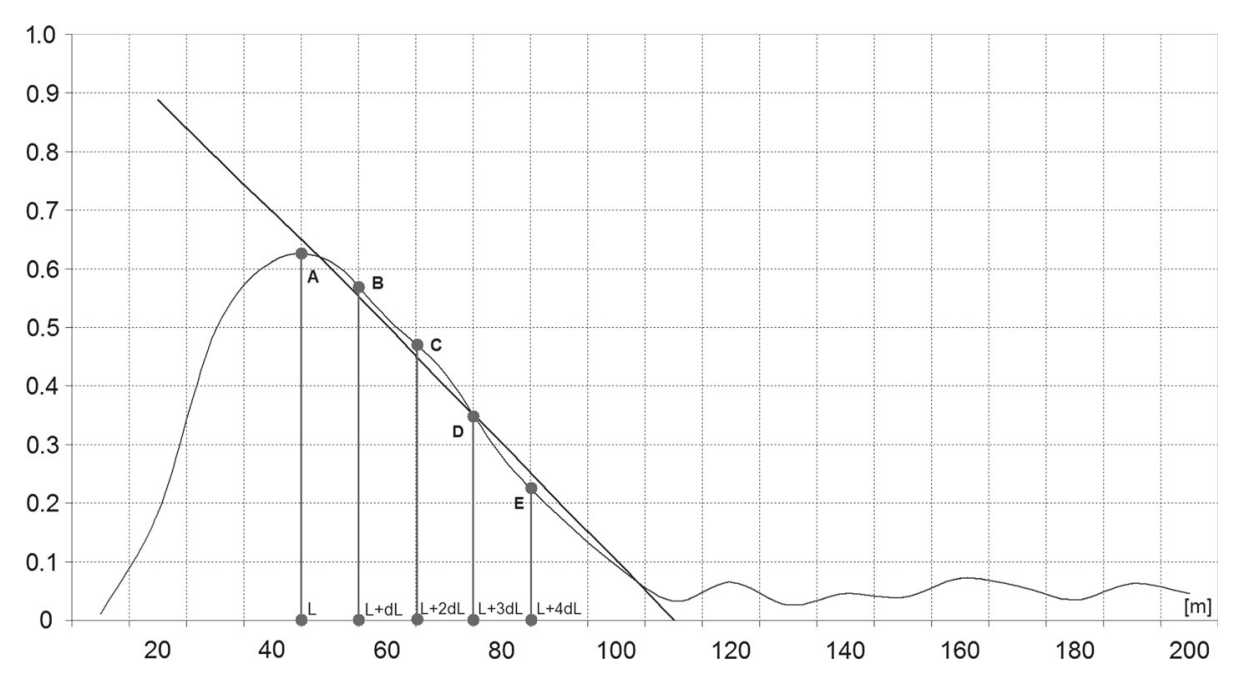

Fig. 4 Calculation of the slope of the autocorrelation peak

common axis. Assumption is made, that the resulting slope of the fitting line represents the overall slope of the autocorrelation peak (as Fig. 4 depicts, vertical axis indicates the correlation coefficient value, whereas the horizontal axis informs what correlation distance is considered onwards). Finally, the obtained values are compared to the reference atmospheric visibility factors, as to calibrate the device.

\subsection{Reference atmospheric visibility calculation}

The standard visibility $V_{n}$ is defined in relation to the distance $R$ for which the threshold of the human discrimination is estimated for the contrast value of $\mathrm{k}_{\min }=0.02$ (Frank et al. 2004). According to Koschmieder (1924), the contrast formula (1) is as follows:

$$
\mathrm{k}=\exp \left(-\frac{\mathrm{bR}}{\mathrm{V}_{\mathrm{n}}}\right)
$$

Components of the equation state, the parameter $\mathrm{b}$ is dependent on $\mathrm{k}_{\min }$; solution for $\mathrm{k}_{\min }=$ 0.02 and $\mathrm{R}=\mathrm{V}_{\mathrm{n}}$ results in parameter $\mathrm{b}$ equal to 3.912 .

The Eq. (1) can also be represented by:

$$
\mathrm{k}[\mathrm{dB}]=10 \log (\mathrm{k})=-4.343 \frac{\mathrm{bR}}{\mathrm{V}_{\mathrm{n}}},
$$

Substituting accordingly (Eq. (2), $\mathrm{b}=3.912$ ):

$$
\mathrm{V}_{\mathrm{n}}=-16.99 \frac{\mathrm{R}}{\mathrm{k}[\mathrm{dB}]}
$$

the formula (3) for standard visibility $\mathrm{V}_{\mathrm{n}}$ can be obtained.

Concerning assessment of a reference atmospheric visibility, utilized method for obtaining of the actual $\mathrm{k}[\mathrm{dB}]$ parameter, is the analysis of visual data (i.e. still frame from a digital camera (Babari et al. 2011; Hautiere et al. 2008)). The irradiance of the captured picture is determined and its distribution along any of the preferred profiles (crosshair selection) is represented on the same picture (consider output presented on the Fig. 5). Analyzing further, 


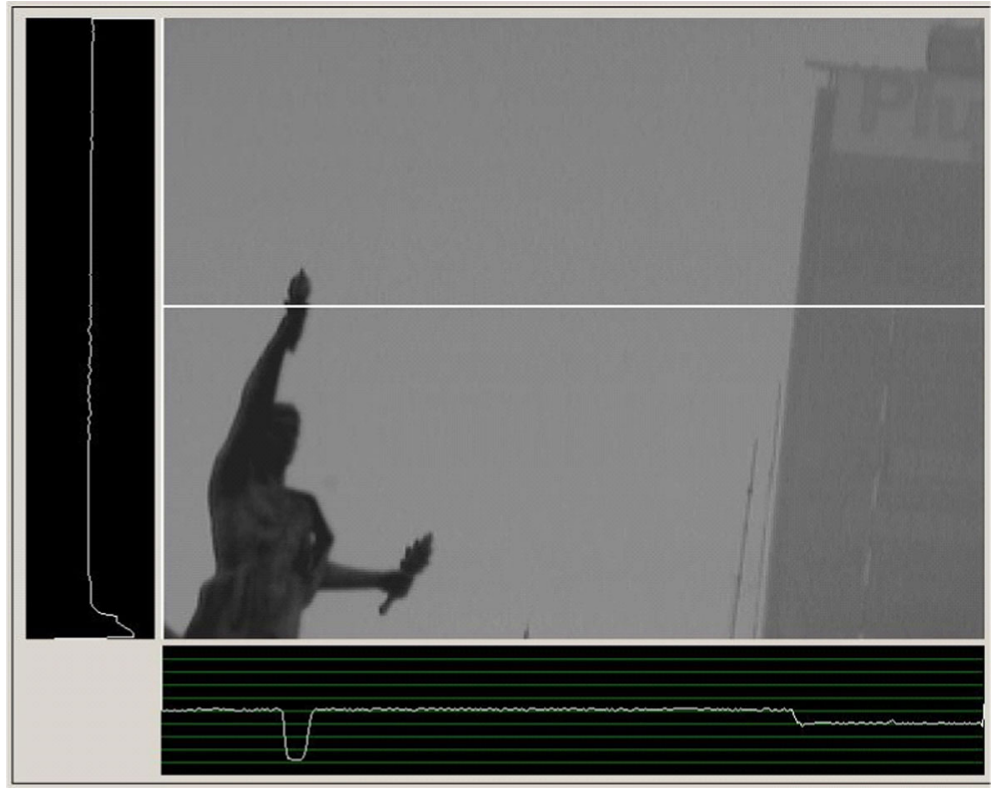

Fig. 5 Contrast estimation for a specific foggy scenery

the contrast level of the dark object, located over the known distance $\mathrm{R}$ is estimated (Holejko et al. 2013). Figure 5 depicts exemplary scenario, where the input parameters are: $\mathrm{R}=1.2 \mathrm{~km}$ and $\mathrm{k} \approx 0.18$, thus the actual standard visibility is approximated on the level of $\mathrm{V}_{\mathrm{n}} \approx 2.7 \mathrm{~km}$.

\section{Results and discussion}

For the final clause, concerning results discussion, as plotted on the Fig. 6, relationship between reference atmospheric visibility and autocorrelation peak is depicted. The vertical axis represents the slope coefficient (i.e. the angular tilt) indicating atmospheric visibility assessed by autocorrelation analysis. As one can observe, the steepness of the characteristic resides upon the approximate range of $\mathrm{V}_{\mathrm{n}}=100 \mathrm{~m}$ towards $1,200 \mathrm{~m}$. Therefore, the plausible accuracy and measurement performance of the device might be reported on such defined distance interval. Next, in order to assess the link between the reference atmospheric visibility $\left(\mathrm{V}_{\mathrm{n}}\right)$ and the relevant slope of the autocorrelation peak $(\rho)$ one can position following equation:

$$
V_{n}=0.739 \exp (-0.00373 \rho)+0.092 \exp (-0.00052 \rho)
$$

The numerical approach of obtaining coefficients in (4) is given by the optimal curve fitting algorithm of the dual-term exponential approximation (MathWorks Inc. MATLAB 2013). As presented on the Fig. 6, where positioning of data fitting curve and measurement outcomes is achieved, the theoretical range of the atmospheric visibility assessment of the designed sensor is limited towards the vicinity of the $3,500 \mathrm{~m}$ marker. However, the reliable interval of estimation is, as previously mentioned, spanned approximately on the range from 100 to $1,200 \mathrm{~m}$. 


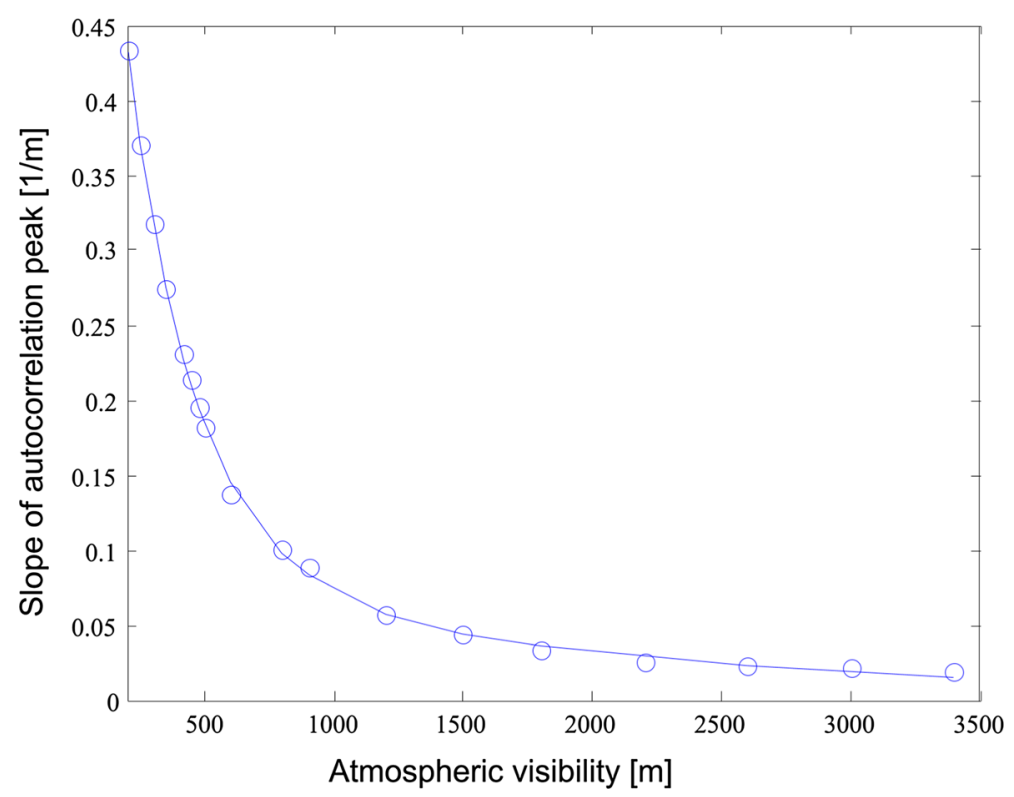

Fig. 6 Slope of the autocorrelation peak versus the reference atmospheric visibility (circle) positioned with the fitting curve (solid line)

\section{Conclusion}

Presented design is based on the detection of backscattered optical signals, that further utilizes correlation coding technique to assess the atmospheric visibility. By series of experimental measurements, as demonstrated in the article, evaluation of the performance of the designed device is stated. Investigation indicates the overall superiority of the correlation coding method applied in evaluation of discussed parameter $\left(\mathrm{V}_{\mathrm{n}}\right)$. Hence, the possibility of assessing the atmospheric visibility over the range (as the numerical spatial parameter, not only as a single point figure) is achieved. What is more, pioneered solution is able to determine the $V_{n}$ parameter to the extent of $1,200 \mathrm{~m}$, what is obtained with real-time processing speed and appropriate accuracy. The designed device is leading its kind, as it utilizes continuous wave, PRBS sequence signal generator, that if necessary might support extended range of measurements. Further, presented gauge might be characterized as a low complexity, cost effective, lightweight and compact device, suitable for continuous measurements and functional improvements of contemporarily deployed systems. Utilizing only a single light source and an avalanche photodiode receiver, the simplicity in design is achieved (measurement system does not imply additional optical switches, amplifiers nor photodiodes). Additionally, while estimation of the atmospheric visibility is performed, the described apparatus might be simultaneously utilized in the area of cloud base elevation measurement (ceilometer).

Open Access This article is distributed under the terms of the Creative Commons Attribution License which permits any use, distribution, and reproduction in any medium, provided the original author(s) and the source are credited. 


\section{References}

Babari, R., Hautiere, N., Papelard, J.: Computer vision for the remote sensing of atmospheric visibility. In: IEEE international conference on computer vision workshops, pp. 219-226; Barcelona, Spain, 6-13 Nov 2011

Babari, R., Hautiere, N., Dumont, E., Paparoditis, N.: A model-driven approach to estimate atmospheric visibility with ordinary cameras. Atmos. Environ. 45(30), 5316-5324 (2011)

Frank, F., Caimi, M., Kocak, D.M., Justak, J.: Remote visibility measurement technique using object plane data from digital image sensors. In: IEEE international geoscience and remote sensing symposium IGARSS '04, vol. 5, pp. 3288-3291; Anchorage, USA, 20-24 Sep 2004

Hautiere, N., Aubert, D., Dumont, E., Tarel, J.-P.: Experimental validation of dedicated methods to in-vehicle estimation of atmospheric visibility. IEEE Trans. Instrum. Meas. 57(10), 2218-2225 (2008)

Holejko, K., Czarnecki, T., Perlicki, K.: Infrared sensor to predict atmospheric visibility and rainfall rate. Int. J. Electron. Telecommun. 59(2), 109-112 (2013)

Kazovsky, L.G.: An optical rain gauge based on forward scattering. IEEE Trans. Geosci. Remote Sens. GE-23(2), 124-131 (1985)

Kemp, M., Kruger, A., Niemeier, J.: Design considerations and signal processing for a microwave rain gauge sensor. In: IEEE international conference on electro/information technology, pp. 1-4; Indianapolis, USA, 6-8 May 2012

Koschmieder, H.: Theorie der horizontalen Sichtweite, Beitr. Phys. fr. Atm. 12, 33-55 and 171-181 (1924)

MathWorks MATLAB: Curve fitting toolbox-linear and nonlinear regression. Documentation center. http:// www.mathworks.com/products/datasheets/pdf/curve-fitting-toolbox.pdf. Accessed 29 May 2013

Siikamaki, R.: New product family of present weather detectors and visibility sensors. Vaisala News 164, 32-33 (2004)

Taillade, F., Belin, E., Dumont, E.: An analytical model for backscattered luminance in fog: comparisons with Monte Carlo computations and experimental results. Meas. Sci. Technol. 19, 055302 (2008) 